\title{
UM ESTUDO DE CASO SOBRE SOCIOEDUCACIONAIS INTEGRAÇÃO DAS FAMÍLIAS DE IMIGRANTES EM GRANADA (ESPANHA)
}

\author{
ESTUDIO DE CASO SOBRE LA INTEGRACIÓN SOCIO-EDUCATIVA DE \\ FAMILIAS INMIGRANTES EN GRANADA (ESPAÑA)
}

\section{A CASE STUDY ABOUT SOCIOEDUCATIONAL INTEGRATION OF IMMIGRANT FAMILIES IN GRANADA (SPAIN)}

\author{
Marina GARCÍA-CARMONA ${ }^{1}$ \\ Alfonso CONDE LACÁRCEL ${ }^{2}$
}

\begin{abstract}
RESUMO: Integração sócio-educativa das famílias imigrantes é um dos pilares de uma sociedade multicultural e multiétnica, gerando oportunidades para todas as pessoas. $\mathrm{O}$ estudo apresentado foi realizado em um bairro suburbano da cidade com forte acolhimento de imigrantes de Granada (Espanha) e tem como objetivo comparar o atual quadro legislativo com os recursos que os pais estrangeiros podem encontrar disponíveis para promover adequada participação nas escolas de seus filhos. Para isso, foram realizadas entrevistas qualitativas de pesquisa com famílias de imigrantes, observações de campo e análise de documentos de tanto as regras regulamentares de base em matéria de imigração como o oferecido por outros atores sociais. Os principais resultados mostram que as famílias de imigrantes recebem aceitação social adequada, apesar das várias dificuldades encontradas. No entanto, falta melhor informação às famílias e grupos de estudantes imigrantes para evitar bolsas de exclusão dentro do centro e um maior envolvimento e compromisso por professores e famílias é necessário dentro de associações de pais de alunos, a partir das principais organizações que envolvem famílias.
\end{abstract}

PALAVRAS CHAVE: Imigração. Integração social e educacional. Educação. Envolvimento da família.

RESUMEN: La integración socioeducativa de las familias inmigrantes es uno de los pilares de una sociedad multicultural, multiétnica y generadora de oportunidades para el conjunto de las personas. El estudio presentado ha sido realizado en un barrio suburbano de fuerte acogida inmigrante de la ciudad de Granada (España) y tiene como objetivo comparar el marco legislativo actual con los recursos que pueden encontrar a su disposición los progenitores extranjeros para favorecer una adecuada incorporación y participación en los centros educativos de sus hijos e hijas. Para ello se ha realizado una investigación de carácter cualitativo con entrevistas a familias inmigrantes, observaciones de campo y una revisión documental, tanto de la normativa básica reguladora respecto a inmigración como la ofrecida por otros agentes sociales. Los principales resultados muestran que las familias inmigrantes perciben una adecuada acogida social a pesar de las distintas dificultades encontradas. Sin embargo a nivel educativo es necesaria una mejora de la información proporcionada a las familias del alumnado inmigrante para evitar posibles focos de exclusión dentro del mismo centro y una mayor implicación y compromiso por parte del profesorado y las familias dentro de

${ }^{1}$ Universidad de Granada (España).

${ }^{2}$ Universidad de Granada (España). 
las asociaciones de madres y padres de alumnado, como organizaciones principales de participación de las familias.

PALABRAS CLAVE: Inmigración. Integración socioeducativa. Educación. Participación familiar.

ABSTRACT: The socio-educational integration of immigrant families is one of the pillars of multicultural, and multiethnic society and it generates opportunities for all. The study presented was conducted in a suburban neighborhood of strong immigrant reception in the city of Granada (Spain), and it seeks to compare the current legislative framework with the resources that immigrant families can find available to encourage an adequate incorporation to schools and their participation in the education of their children. A qualitative approached was used with interviews to immigrant families, field observations and a literature review. The results highlight a suitable host despite the difficulties found. However, regarding the educational context, it is necessary to improve the information for parents to avoid exclusion within the school. Also a greater involvement and commitment of teachers and families within parents and teachers associations as main organizations for family participation is needed.

KEYWORDS: Immigration. Social and educational integration. Education. Family participation.

\section{Introducción}

Una de las características históricas del último tercio del siglo XX y comienzos del XXI, es la existencia de grandes desplazamientos de población entre distintos países (BENÍTEZ ROBREDO et al., 2004). A lo largo de estos años, la acogida de personas procedentes de otras fronteras, ha ido sufriendo diferentes fluctuaciones: de momentos de gran afluencia a Europa y a España en concreto, como miembro de la Unión Europea, por las "bondades de una vida mejor", se ha ido produciendo una paulatina disminución de la llegada de inmigrantes desde el comienzo de la crisis económica en 2009. Este hecho lo podemos observar en la Colección de Documentos del Observatorio Permanente de la Inmigración del Ministerio de Trabajo e Inmigración (2009) o en trabajos de investigación como los de Bedmar y Caro (2013) y Cábala (2000).

El presente estudio pretende dar una visión de la realidad social actual en referencia a las facilidades de acceso a los diferentes recursos por parte de la población inmigrante cuando llega al país de acogida (España), su integración social y las posibles influencias que puedan tener sobre los aspectos educativos de sus hijos e hijas. La atención se centró en la acogida a la llegada a España, su permanencia y acceso a los distintos servicios y fundamentalmente en su repercusión final para aquellos padres y madres inmigrantes sobre la educación de sus hijos e hijas. Ésta variará en función de que 
los progenitores posean una situación regularizada (con contrato de trabajo desde origen, con autorización por estudios o investigación, motivos humanitarios...) o la llegada se produzca de manera irregular e indocumentada (BENÍTEZ ROBREDO et al., 2004). Destaca en esta línea la información del Defensor del Pueblo Andaluz en conjunción con otras organizaciones que trabajan en esta área, además de experiencias relacionadas con la participación de las familias inmigrantes en los centros educativos (PONS, ROVIRA y PEDRO, 2012; CIVIS y LONGAS, 2015).

De acuerdo con el R.D. 2393/2004, de 30 de Diciembre, por el que se aprueba el Reglamento de la Ley Orgánica 4/2000, de 11 de Enero sobre Derechos y Libertades de los Extranjeros en España y su Integración Social, que incluye todas las modificaciones de la Ley hasta 5 de Agosto de 2010, tanto la entrada y salida, como la estancia y residencia, se hallan fuertemente burocratizadas. Nos encontramos con unos plazos en teoría suficientemente amplios y claros para que cualquier persona de manera "normalizada" desde el país de origen, pueda acceder a los recursos que dispone el país de acogida cuando llega.

Igualmente, se prevén las normas de asilo para casos humanitarios con el fin de responder a las necesidades de la realidad cambiante y sus destinatarios, tras llegar a un consenso entre los distintos agentes sociales. ¿Pero qué ocurre con la inmigración irregular y que no dispone de los documentos necesarios para regularizar su estancia y que desean acceder a recursos básicos y fundamentales como la educación? En este sentido, múltiples asociaciones les ayudan a regularizar su situación facilitándoles toda la información posible, así como ayuda especializada. Por ejemplo, podemos citar la Guía para personas sin papeles, elaborada por la Asociación APDHA (Asociación Pro Derechos Humanos de Andalucía) de 2011, con la colaboración del Defensor del Pueblo Andaluz y otras ONGs.

En todos los casos la concesión a una persona extranjera de la posibilidad de trabajar y de que sea residente en España, se vincula a un largo procedimiento administrativo por parte de la autoridad laboral autonómica competente y a otro procedimiento estatal sobre la estimación o denegación de que el extranjero resida en mencionado país. Pero, ¿cómo les afecta a las familias inmigrantes y a sus hijos e hijas en referencia al acceso y uso del sistema educativo del país de acogida?, ¿se sienten las familias inmigrantes respaldadas para participar en las actividades relacionadas con la educación de sus hijos e hijas propuestas por el centro escolar? 
Las investigaciones realizadas al respecto sobre las familias y el desarrollo socioeducativo de los hijos e hijas (SANTOS REGO, 2008; SANTOS REGO y LORENZO MOLEDO, 2009; SOCIAS, BALLESTER Y MARCH, 2013), entre otras, analizan entre distintos aspectos la procedencia de las familias inmigrantes en relación a la participación en los centros. Desde esta perspectiva se nos presenta cómo afecta la cultura del país de procedencia y los posibles prejuicios o sesgos tanto por parte de los inmigrantes como del país de recepción (YESTE, CASABONA y COMPTE, 2013).

Por otro lado, si nos centramos en la legislación vigente, encontramos que las madres y padres extranjeros que deseen realizar trabajos de investigación o formación no remunerados laboralmente o cursar o ampliar estudios, deben disponer del correspondiente visado de estudios, debiéndose cumplir una serie de requisitos que, en muchas ocasiones, conllevan un largo período de tiempo y, en otras, terminan en un abandono del inmigrante puesto que tienen miedo a que los descubran y tengan que volver a su país (GARRETA, 1994).

Finalmente debemos tener en cuenta que las Administraciones Educativas, en el ejercicio de sus competencias en materia de educación, podrán facilitar el acceso de los extranjeros menores de edad que se hallen empadronados en un municipio a los niveles de enseñanza posobligatoria no universitarios y a la obtención de la titulación académica correspondiente en igualdad de condiciones que los españoles de su edad. En este sentido, el Ministerio de Trabajo e Inmigración (2008) señala que los retos más destacados a los que la educación en España se enfrenta para la integración del alumnado extranjero son la garantía de su incorporación con plenitud de derechos y deberes de ciudadanía, el desarrollo de procesos de gestión educativa desde el respeto a la diversidad y la igualdad de oportunidades, la oferta de nuevas respuestas educativas como métodos organizativos y pedagógicos basados en los principios de la educación intercultural y el mayor aporte de recursos humanos y económicos.

Asimismo la educación intercultural en los centros educativos se presenta como reto en las organizaciones escolares, junto con la atención a las buenas prácticas que se hacen imprescindibles (MESA y SOLBES, 2012; FRUTOS, 2013; IZQUIERDO, 2015). La comunicación con las familias y su participación activa en los centros educativos, como puede ser el caso de las asociaciones de madres y padres de alumnado (AMPA), proporcionan un contexto único de interacción de las familias de origen inmigrante y las autóctonas, además de ser un recurso óptimo de inclusión social (GARCÍA-CARMONA, 2014). 
De este modo, los objetivos perseguidos en esta investigación son: a) Ofrecer una visión comparativa entre la legislación actual sobre inmigración y la realidad percibida y vivida por los padres y madres inmigrantes con respecto a su integración en el país de acogida, b) Conocer las experiencias de participación de los padres y madres inmigrantes en la escuela de sus hijos e hijas y su educación c) Investigar las expectativas de futuro de las familias inmigrantes.

\section{Metodología}

Stake (2005, p.11) define el estudio de caso como: "el estudio de la particularidad, [...] la complejidad de un caso singular, para llegar a comprender su actividad en circunstancias." Trata de analizar en profundidad una realidad, suceso o programa en un individuo o grupo con el fin de obtener una descripción detallada que nos ayude a solucionar problemas prácticos. Igualmente, debemos resaltar las ventajas del uso socioeducativo de los estudios de caso de Latorre y colaboradores (1998) que señalan que:

$\checkmark$ Puede ser una manera de profundizar en un proceso de investigación a partir de unos primeros datos analizados.

$\checkmark$ Es apropiado para investigaciones a pequeña escala, en un marco limitado de tiempo, espacio y recursos.

$\checkmark$ Es un método abierto a retomar otras condiciones personales o instituciones diferentes.

$\checkmark$ Es de gran utilidad para el profesorado que participa en la investigación. Favorece el trabajo cooperativo y la incorporación de distintas ópticas profesionales a través del trabajo interdisciplinar; además, contribuye al desarrollo profesional.

$\checkmark$ Lleva a la toma de decisiones, a implicarse, a desenmascarar prejuicios o preconcepciones...

Nuestro estudio se adapta a este tipo de metodología de investigación que proporciona numerosos beneficios a la hora de interpretar los resultados obtenidos. El contexto en el que se ha llevado a cabo la investigación es el barrio de La Chana de la ciudad de Granada. Se trata de un barrio que surgió a lo largo del siglo XX, como barrio dormitorio del extrarradio que acogía a la población procedente de zonas rurales y que posteriormente fue asentándose y creciendo. A finales del siglo XX y principios de este siglo XXI, la población predominante, de clase obrera, empezó a recibir población inmigrante, siendo hoy día una parte importante de la vecindad. El centro escolar seleccionado está situado cerca de una entidad de carácter religioso que colabora en 
actividades de acogida del inmigrante y posee un alto porcentaje de alumnado de diferentes países.

Por este motivo, el instrumento de recogida de datos utilizado en la investigación ha sido la entrevista ya que permite obtener información sobre acontecimientos y aspectos subjetivos de las personas: creencias y actitudes, opiniones, valores o conocimiento, que de otra manera no estarían al alcance del investigador (ARNAL et al., 1995, p. 307). La finalidad principal de la entrevista es que el investigador sea capaz de encontrar lo que es significativo para los informantes, descubrir acontecimientos y dimensiones subjetivas de las personas como los valores, pensamientos, etc. (COLÁS, 2001, p. 275). En esta ocasión se ha optado por una modalidad de entrevista semi-estructurada que ha permitido a los sujetos entrevistados tener una cierta libertad a la hora de expresar sus vivencias.

Por otro lado, siguiendo a Del Rincón y colaboradores (1995, p. 324), para la planificación de las entrevistas se ha tenido en cuenta las siguientes fases:

$1^{\circ}$. Objetivo general de la entrevista: averiguar las trayectorias de vida de los inmigrantes entrevistados teniendo en cuenta las influencias de la legislación vigente y la acogida inmigrante sobre todo en el ámbito de la educación.

$2^{\circ}$. Muestreo de personas a entrevistar: se ha llevado a cabo un muestreo opinático de informantes clave.

$3^{\circ}$. Desarrollo de la entrevista: las entrevistas semi-estructuradas se desarrollaron a lo largo de los primeros meses del curso escolar; los meses anteriores fueron empleados en una exhaustiva revisión documental sobre la normativa más actualizada con el fin de establecer una base de análisis y comparativa con la realidad lo más objetiva posible.

El hecho de utilizar entrevistas semi-estructuradas con un guión preestablecido pero abierto, se ideó con el fin de poder enriquecer el discurso biográfico narrativo de las personas entrevistadas y obtener la máxima información posible. Teniendo en cuenta estos aspectos, se han llevado a cabo 10 entrevistas a madres y padres inmigrantes, con una duración media de 45 minutos, en función de cada una de las personas entrevistadas. Se les planteó un conjunto de 18 preguntas, así como unos datos identificativos previos, sobre su procedencia, sexo, situación familiar, laboral, nivel de estudios y edad, y fueron desarrolladas en espacios del centro educativo. La entrevista elaborada y validada al efecto fue la siguiente: 


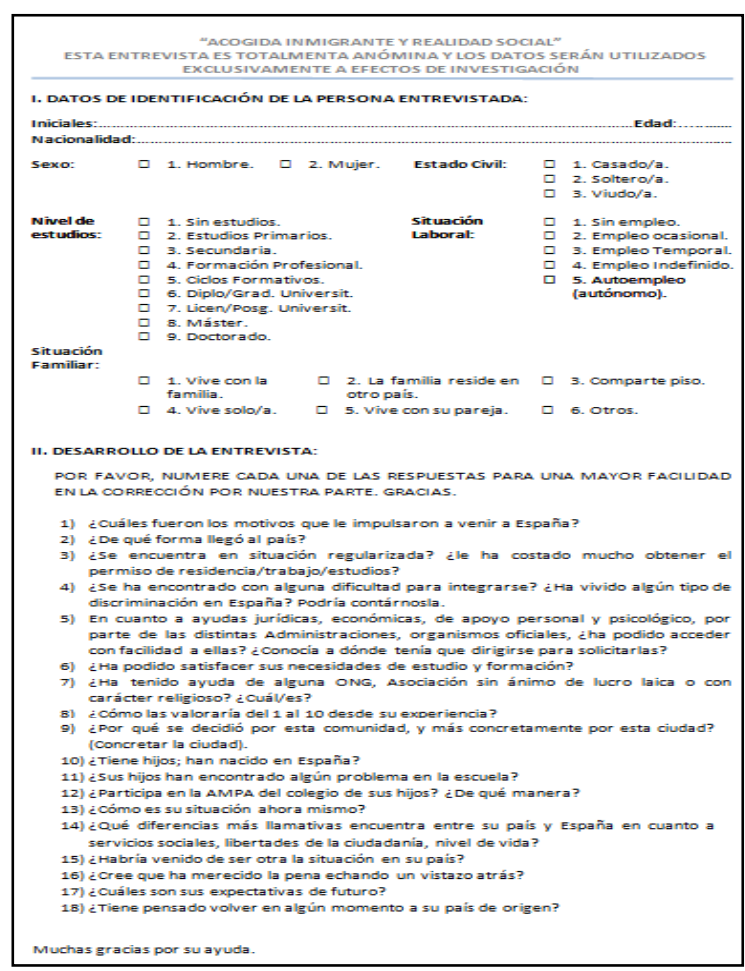

Fuente: Elaboración propia.

\section{Resultados y discusión}

Una vez examinados los datos obtenidos con el programa informático Nvivo8, hemos podido establecer una serie de intereses fundamentales según nuestros objetivos de investigación, entre los que se puede apreciar una clara priorización de determinados bloques de preguntas, que han influido en el número de referencias, frases y palabras obtenidas. Además, se ha realizado un análisis de contenido de las entrevistas realizadas (BARDIN, 1986). Principalmente en aquellos aspectos más significativos referidos a la incorporación de estas familias a la sociedad española con todos sus deberes y derechos, además de los elementos que, a juicio de los entrevistados, favorecen la incorporación a la comunidad escolar, su información y participación y una educación de calidad a sus hijos e hijas.

De acuerdo con las distintas dimensiones trabajadas en cada uno de los bloques en los cuales hemos dividido la entrevista (Bloque I: grado de integración en el país; Bloque II: aspectos educativos; Bloque III: expectativas de futuro), encontramos en el Bloque I, un mayor número de referencias centradas en distintos aspectos de la acogida y su incorporación a la sociedad española, con respecto a otros bloques. 


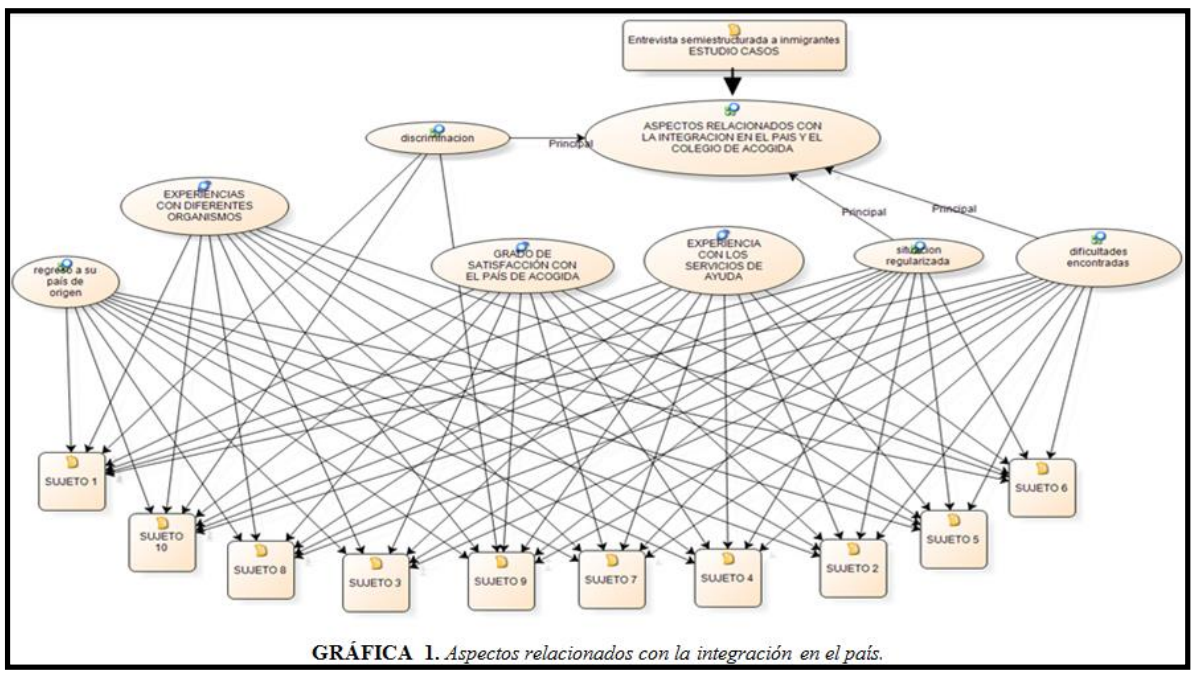

Fuente: Elaboración propia.

A continuación en la Tabla 1 presentamos por frecuencia de respuestas los resultados obtenidos, así como los modelos generados por el programa para una mayor comprensión:

Tabla 1. Grado de integración en el país.

\begin{tabular}{|c|c|c|}
\hline \multicolumn{3}{|c|}{ Grado de satisfacción con el país de acogida } \\
\hline 45 referencias & 49 frases & 403 palabras \\
\hline \multicolumn{3}{|c|}{ Situación regularizada } \\
\hline 36 referencias & 36 frases & 308 palabras \\
\hline \multicolumn{3}{|c|}{ Dificultades encontradas } \\
\hline 16 referencias, & 17 frases & 285 palabras \\
\hline \multicolumn{3}{|c|}{ Experiencia con los servicios de ayuda } \\
\hline 13 referencias, & 14 frases & 196 palabras \\
\hline \multicolumn{3}{|c|}{ Regreso a su país de origen } \\
\hline 18 referencias & 16 frases & 182 palabras \\
\hline \multicolumn{3}{|c|}{ Discriminación } \\
\hline 16 referencias & 4 frases & 92 palabras \\
\hline \multicolumn{3}{|c|}{} \\
\hline 4 referencias &
\end{tabular}

Fuente: Elaboración propia.

Como se puede apreciar en la Tabla 1, el grado de satisfacción general con el país de acogida es el tópico más comentado, seguido de la situación de regularización final y 
las distintas dificultades encontradas a la hora de obtener información sobre el proceso a seguír, los requisitos para la nacionalidad, o el acceso a recursos sociales fundamentales. En menor medida es expresado, el trato recibido y la ayuda por parte de los distintos servicios administrativos y organimos a los que han podido acceder. Como contrapunto positivo, podemos apreciar una escasa referencia en esta experiencia de vida; a cualquier tipo de discriminación vivida al llegar a España.

Respecto a los aspectos relacionados con la educación, encontramos que en este sentido los padres y madres no han tenido problemas de participación en el centro educativo donde matricularon a sus hijos e hijas, percibiendo una acogida favorecedora de la integración socio-educativa. En general han encontrado las puertas abiertas de los centros educativos y han percibido un clima de bienvenida. Las ayudas a nivel educativo, pasaron principalmente por adaptaciones no significativas del curriculum en base a las posibles carencias o dificultades idiomáticas que pudiesen existir en algunos niños y niñas.

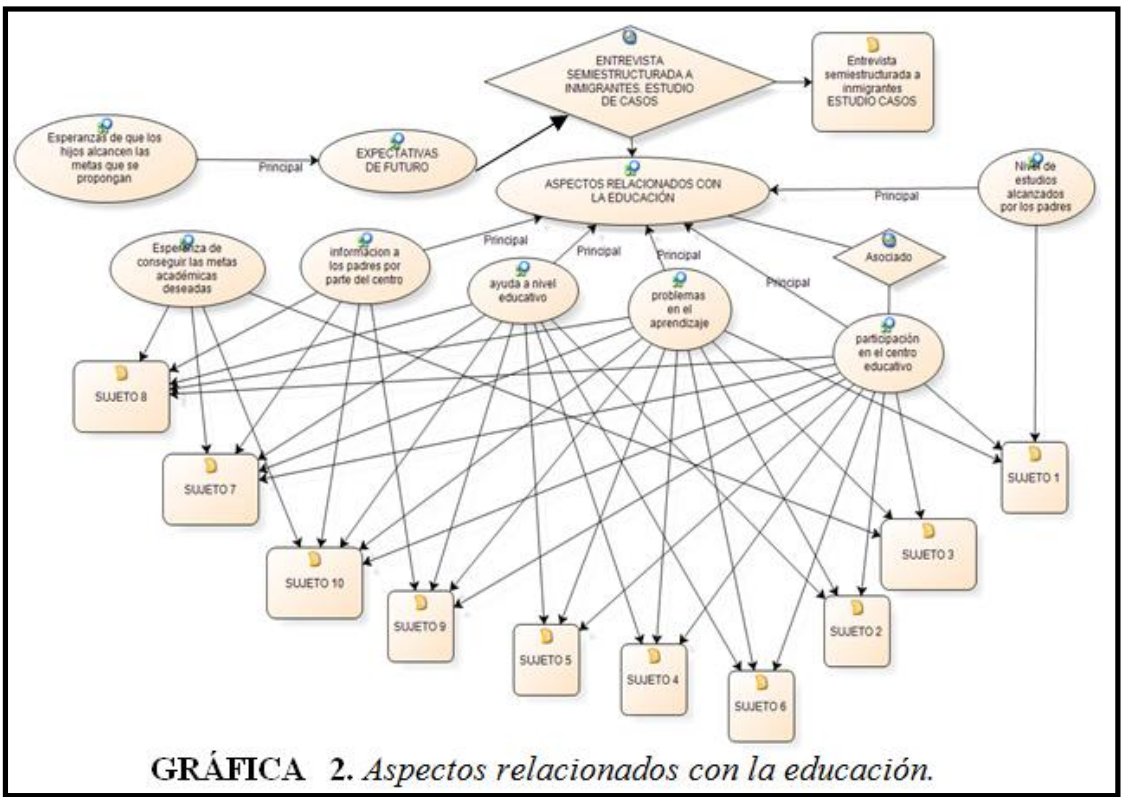

Fuente: Elaboración propia.

Como se puede apreciar en la Tabla 2, la frecuencia o mayor interés respecto al bloque referido a los aspectos educativos podemos encontrarla en la información proporcionada a los padres y madres en los centros educativos; seguida de las ayudas que pueden ofrecerles a sus hijos e hijas, los problemas en el aprendizaje que pudiesen surgir y la participación en el centro educativo. Estos cuatro aspectos serán decisivos para 
mejorar la experiencia inclusiva de las familias extranjeras en el centro educativo y en la comunidad.

Tabla 2. Aspectos educativos.

\begin{tabular}{|c|c|c|}
\hline \multicolumn{3}{|c|}{ Información a los padres/madres por parte del Centro } \\
\hline 6 referencias & 7 frases & 129 palabras \\
\hline \multicolumn{3}{|c|}{ Problemas en el aprendizaje } \\
\hline 10 referencias & 10 frases & 118 palabras \\
\hline \multicolumn{3}{|c|}{ Ayuda a nivel educativo } \\
\hline 10 referencias & 11 frases en centro educativo \\
\hline \multicolumn{2}{|c|}{ Nivel de estudios alcanzado por los padres/madres } \\
\hline 12 frases & 10 frases & 88 palabras \\
\hline 10 referencias & 4 frases & 77 palabras \\
\hline \multicolumn{2}{|c|}{ Esperanzas de que los hijos/as alcancen las metas que se propongan } \\
\hline 4 referencias & 4 frases & 92 palabras \\
\hline \multicolumn{3}{|c|}{ Esperanzas de conseguir las metas académicas deseadas } \\
\hline 4 referencias & .
\end{tabular}

Fuente: Elaboración propia.

En el bloque siguiente referido a las expectativas de futuro y que podemos ver tanto en la Gráfica 3 como en la Tabla 3 con los resultados correspondientes, el interés principal se encuentra en la convivencia y vida familiar seguida de la oferta laboral adecuada. En este sentido, y a pesar de estar en primera posición las esperanzas de las familias en que sus hijos e hijas se incorporen con facilidad a la nueva sociedad y sistema educativo, paliando todas las posibles dificultades educativas, pudimos apreciar la preocupación tanto en madres como padres de conseguir un buen trabajo que les permita cubrir todas sus necesidades. 


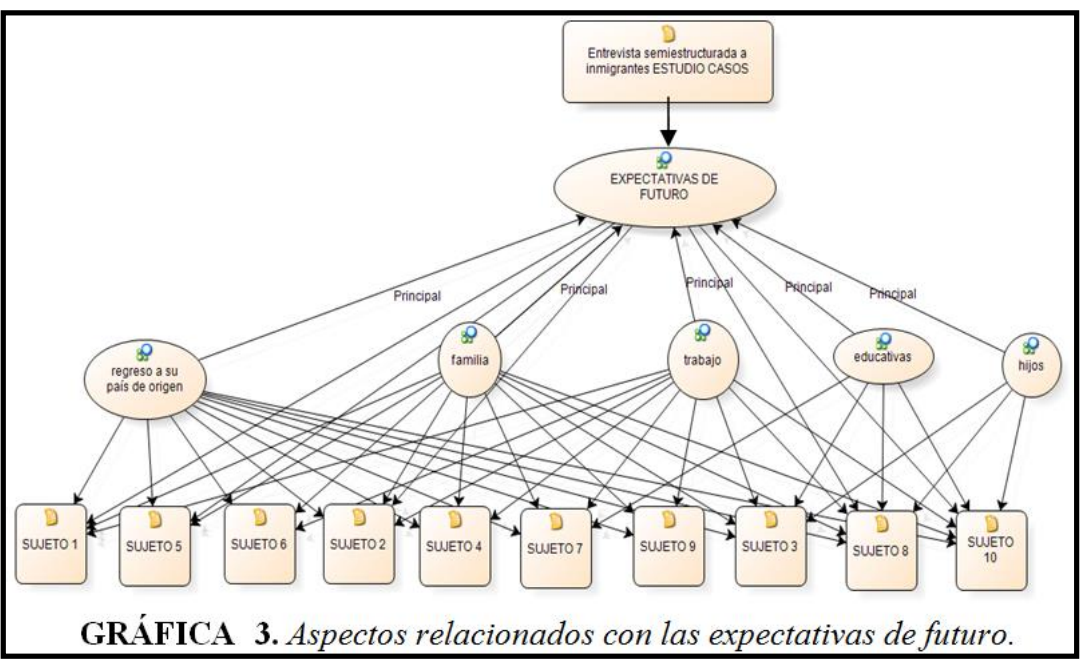

Fuente: Elaboración propia.

Tabla 3. Expectativas de futuro.

\begin{tabular}{|c|c|c|}
\hline \multicolumn{3}{|c|}{ Esperanzas relacionadas con la convivencia y vida familiar } \\
\hline 17 referencias & 17 frases & 191 palabras \\
\hline \multicolumn{3}{|c|}{ Esperanzas de obtener una ocupación laboral adecuada } \\
\hline 15 referencias & 15 frases & 143 palabras \\
\hline \multicolumn{3}{|c|}{ Expectativas de futuro } \\
\hline 8 referencias & 8 frases & 106 palabras \\
\hline
\end{tabular}

Fuente: Elaboración propia.

Una vez realizado el análisis de contenido de las entrevistas se obtiene una visión de la situación que han vivido y están viviendo las familias inmigrantes de los estudiantes. Autores como Terrén y Carrasco (2007) han llevado a cabo un análisis sobre la familia, la emigración y cómo las expectativas y experiencias influyen sobre el alumnado y la situación escolar. En este sentido, observamos que la llegada al país ha sido debida a motivos de trabajo o familiares, y en la mayoría de los casos de forma ilegal en lo referente a la entrada, pero sin demasiadas complicaciones por lo expresado por los entrevistados.

La dificultad a la hora de legalizar su situación ha sido una constante que se ha repetido en todos los casos estudiados: Estoy en trámites aún, mi mujer si está regularizada. Es una situación muy incómoda porque se necesita para hacer cualquier cosa (sujeto 10).

Por otra parte, y a pesar de que muchas de ellas hacen referencia a las múltiples dificultades que han encontrado a la hora de establecerse y acceder a los servicios públicos estatales (sanidad, educación, regularización de su situación...), se puede apreciar un alto grado de satisfacción de las familias inmigrantes respecto a España: Sí, 
sobre todo por el bienestar de mis hijos (sujeto 8). Se observa que un aspecto que se repite en diferentes entrevistas es la dificultad de convalidar sus titulaciones y de acceder a cursos formativos, encontrándose de este modo con que en España no tienen ningún logro académico. A este aspecto se le suma también la dificultad para obtener el visado de estudios.

También destacan la buena disposición del resto de organizaciones no lucrativas, organizaciones no gubernamentales y servicios de ayuda, y los intentos por facilitarles la inclusión en nuestra sociedad:

Sí, en todas incluso para buscar un abogado, salud, alimentación. Dan una cita con la visitadora y ella ve lo que hace falta. Preguntando sabía dónde dirigirme. Al principio me daba miedo ir al médico, en Bolivia es muy caro y "se vende". Aquí es más accesible (sujeto 10).

Las situaciones de discriminación que han podido padecer han sido muy poco significativas ya que los sujetos entrevistados han tenido una experiencia positiva con la relación con las personas y raramente se han enfrentado a momentos de aislamiento social, en base a lo que han respondido. Sin embargo, a pesar de mostrar una actitud positiva hacia el país de acogida, la mayoría de las personas entrevistadas no descartan volver a su país, pero a muy largo plazo y de vacaciones por el momento: Puede que de aquí a unos años vaya de visita pero definitivamente no se sabe todavía (sujeto 9).

Respecto a los aspectos educativos, lo más valorado en este grupo de cuestiones planteadas ha sido el hecho de tener una información por parte del centro sobre la evolución de sus hijos/as y los posibles problemas que pudiesen tener: Hace 2 meses puse una querella, en el cambio de curso. En $1^{\circ} B$ eran todos españoles, mientras que en el otro curso principalmente marroquíes, de Europa del este y no se hablaba apenas el castellano, y yo quería que mi hija aprendiese la lengua castellana. Por lo demás, ningún problema (sujeto 1). Las personas entrevistadas, a pesar de reconocer que obtienen alguna información del centro educativo, insisten en la necesidad de que esa información sea más abundante y continua.

La participación a través de las distintas actividades que ofertan las escuelas, tutorías, AMPA y las ayudas que se ofrecen a nivel educativo al alumnado, han resultado ser un aspecto muy valorado por los padres y madres aunque con algunas mejoras: $\mathrm{He}$ estado los años pasados pero no he visto apenas beneficio, no asisten muchos padres a las reuniones y no estoy satisfecho. Criticaban al director. En Bolivia las reuniones de 
padres son obligatorias y a los niños se les quita un punto si no asisten los padres. Hay una escuela de padres en todos los colegios obligatoria. Todos pertenecen a ella porque se paga una cuota y el AMPA paga a psicólogos y especialistas (sujeto 10). De este modo se puede apreciar cómo estos modelos de participación son un elemento inclusivo de las familias en la escuela y, por tanto, en la comunidad educativa.

Nos gustaría hacer hincapié en este sentido, en la posibilidad de establecer de manera general-, medidas de participación de toda la comunidad educativa de los centros. En especial de la relación entre los padres y madres y profesionales de la educación en nuestra sociedad, ya que en muchas ocasiones, en función del nivel de formación y grado de conciliación laboral y familiar de los primeros, no se realiza de la manera más adecuada para los hijos e hijas debido a una falta de comunicación funcional y de implicación en la medida de las posibilidades de los progenitores en la consecución de las habilidades, competencias u objetivos educativos pretendidos con respecto a la programación de curso e iniciativas de los centros para favorecer la convivencia.

Por otro lado, son interesantes las escasas referencias de los padres y madres a que sus hijos e hijas alcancen las metas que se propongan, tanto vitales como académicas y de futuro. Sin embargo, cuando lo hacen, esperan que consigan los mejores resultados y sean felices: Para mis hijos que estudien, que ellos salgan adelante, con ese privilegio de que estudien lo que quieran (sujeto 3).

Por último, respecto al tercer bloque que alude a las expectativas de futuro, decir que éstas se basan fundamentalmente en las referidas a la convivencia y vida familiar y a la preocupación por encontrar una ocupación laboral adecuada. Podemos encontrar citas como: Para que mis hijos estudien, que ellos salgan adelante, con ese privilegio de que estudien lo que quieran (sujeto 3).

La idea predominante es que los niños y niñas alcancen unas competencias académicas y profesionales que les capaciten para la vida laboral, con un nivel de calidad de vida equilibrado y satisfactorio con, al menos, unos niveles de felicidad mínimos. Los aspectos generales relacionados con el desarrollo vital en otras áreas, no parece apreciarse demasiado.

\section{Conclusiones}

Las experiencias de llegada a España por parte de las familias inmigrantes entrevistadas son muy diversas: matrimonios de conveniencia con una persona nacida 
aquí para conseguir la nacionalidad; con visados falsos comprados en otros países; por motivos de reunificación familiar; con periodos limitados al encuentro de un puesto de trabajo... Todo para alcanzar una seguridad en derechos básicos fundamentales como son el empleo, la sanidad o la educación de sus hijos e hijas que en sus países de procedencia es muy difícil conseguir sin un estatus económico elevado.

Como podemos apreciar, la relación entre normativa legal y realidad social es compleja en la mayoría de los casos entrevistados. El desconocimiento de la información sobre los recursos a los que se puede acceder, junto a determinadas actitudes de desigualdad por no ser del país o estar indocumentados, dificulta la integración social, así como una participación más comprometida y eficaz en los centros educativos: $A l$ principio en el hospital con las enfermeras cuando llegué sí. No tenía papeles y no me quisieron atender (sujeto 4).

Podríamos alegar en este caso, que el estudio realizado por el grupo de trabajo CCCB con Delgado (2003) en el libro Inmigración y Cultura, sigue presente como elemento de revisión de las políticas que se desarrollan en España y otros países de la comunidad europea, endureciéndose el discurso debido a la crisis actual en que nos encontramos y la realidad cercana de las comunidades en las que se produce la integración y la convivencia en distintos grados (BEDMAR y CARO, 2013). En los únicos casos en los cuales, la llegada e incorporación se produce sin demasiados problemas, siempre se encuentran aparejados a un contrato de trabajo o familiares residentes en el país: Vine para trabajar, mi familia tiene un negocio aquí y necesitaban ayuda (sujeto 2).

Si centramos nuestra atención en los aspectos educativos, sería conveniente evaluar los distintos canales utilizados, con el fin de mejorar el sistema de comunicación escuelafamilia y conseguir que sea más eficiente. La mayoría de los sujetos entrevistados parecen desconocer el funcionamiento de los centros educativos en España, así como las posibilidades de participación que existen en el mismo como es el caso de las AMPA. De este modo se hacen imprescindibles medidas que fomenten la presencia de las familias en la escuela y programas formativos que respondan a las necesidades de éstas. Se trata de hacer un currículum funcional con la pretensión de que se produzcan canales bidireccionales de comunicación y colaboración entre los educadores y responsables al cargo de los centros educativos de acogida y las madres y padres preocupados por un desarrollo en igualdad de condiciones, independientemente del país de procedencia (NÍKLEVA, 2012). 
Los hijos e hijas de los sujetos entrevistados no presentan situaciones de dificultades de aprendizaje, en gran medida debido a la pronta incorporación de éstos al colegio (en la etapa de Educación Infantil) y haber recibido una respuesta de calidad por parte del profesorado. La estabilidad en la escolarización desde su inicio hasta la Educación Primaria en el mismo centro, les ha ayudado a integrarse sin complicaciones.

Desde nuestro punto de vista, y en consonancia con otros investigadores como Díaz-Barriga (2007) creemos, además, que es importante tener en cuenta las distintas líneas de investigación psicoeducativa desde las perspectivas de la interacción sociocultural y educativa, que afectan a los procesos de enseñanza aprendizaje directa o indirectamente en el alumnado. De este modo se podrían evitar posibles dificultades de aprendizaje.

Por último, podemos señalar que las expectativas de futuro de los padres y madres entrevistados, variará según los logros y puestos de trabajo obtenidos, así como calidad de vida alcanzada (INTXAUSTI, ETXEBERRIA y JOARISTI, 2014). De las respuestas obtenidas en las entrevistas, se puede percibir un deseo de permanecer en el país de acogida, y la riqueza de la información proporcionada por ellas debe ser tenida en cuenta a la hora de hacer diseños educativos más sensibilizados y adaptados a las personas.

\section{REFERENCIAS}

ARNAL, J; DEL RINCÓN, D; LA TORRE, A. y SANS, A. Técnicas de Investigación en Ciencias Sociales. Barcelona: Dykinson S.L. 1995

\section{ASOCIACIÓN PRO DERECHOS HUMANOS DE ANDALUCÍA (Ed.). Guía para personas sin papeles. 2011 Recuperado de:}

<http://www.apdha.org/index.php?option=com_content\&task=view\&id=871\&Itemid=4 $5>$.

BARDIN, L. Análisis de contenido. Madrid: Akal. 1986.

BEDMAR, V.L, y CARO, M.T.T. Políticas socioeducativas sobre inmigración en la Unión Europea. Revista complutense de educación, 22(2), 211-233. 2012

BEDMAR, V.L., y CARO, M.T.T. Políticas socioeducativas de integración de los inmigrantes en Alemania, España y Francia. Pedagogía social. Revista interuniversitaria, 21, 111-139. 2013. 
BENÍTEZ ROBREDO T, LLERENA ACHÚTEGUIA, P., LÓPEZ GIMÉNEZB, R., BRUGERA MORENOA, C., LASHERAS LOZANOCET, L. Inmigración y determinantes sociales de salud. An Pediatr (Barc), 60(1), 9-15 2004

CÁBALA, S. Estudio sobre la integración de los niños procedentes de la migración escolarizados en Madrid capital. Revista Complutense de Educación, 11(2), 107-147. 2000

CIVÍS ZARAGOZA, M.; y LONGÁS MAYAYO, J. La colaboración interinstitucional como respuesta al desafío de la inclusión socioeducativa. Análisis de 4 experiencias de trabajo en red a nivel local en Cataluña. Educación XX1, 18(1), 213-236. doi: 10.5944/educXX1.18.1.12318 2015

COLÁS, P. Métodos y Técnicas Cualitativas de Investigación en Psicopedagogía. En BUENDIA EISMAN, L. y otros. Métodos de Investigación en Psicopedagogía. Madrid: McGraw - Hill. 2001

COMISIÓN ESPAÑOLA DE AYUDA AL REFUGIADO (CEAR). La situación de las personas refugiadas en España. Informe 2011. Madrid: Entinema. 2011

DE LA TORRE, S. (coord.) Cómo innovar en los centros educativos. Estudio de casos. Madrid: Escuela Española. 1998

DELGADO, M. (ed.) Inmigración y cultura. Barcelona: Centre de Cultura Contemporània de Barcelona (CCCB). 2003

DEL RINCÓN, D.; LA TORRE, A.; ARNAL, J. y SANS, A. Técnicas de Investigación en Ciencias Sociales. Madrid: Dykinson. 1995

DÍAZ-BARRIGA, F. La investigación psicoeducativa en la perspectiva sociocultural: algunas aproximaciones y retos. Revista Sembrando Ideas, $\left(\mathrm{N}^{\circ} 1\right.$ Septiembre) Recuperado de: www.sembrandoideas.cl 2007

FRUTOS, A. La educación intercultural en el contexto social y educativo. En-clave pedagógica, 13. 2013

GARCÍA-CARMONA, M. Análisis de las percepciones sobre liderazgo y participación de las familias en asociaciones de madres y padres en contextos multiculturales. Un estudio comparativo entre Nueva York y Granada. Tesis Doctoral Universidad de Granada. 2014

GARRETA, J. Expectativas educativas y sociales de las familias inmigrantes. Papers. Revista de Sociología, 43, 115-122. 1994

GARRETA, J. Escuela, familia de origen inmigrante y participación. Revista de Educación, 345, 133-155. 2008

INTXAUSTI, N., ETXEBERRIA, F., y JOARISTI, L. ¿Coinciden las expectativas escolares de la familia y del profesorado acerca del alumnado de origen inmigrante? RELIEVE-Revista Electrónica de Investigación y Evaluación Educativa, 20(1). 2014 
IZQUIERDO, R. La atención a la diversidad cultural: el reto de las organizaciones escolares. pp. 189-202. Tendencias Pedagógicas, (9). 2015

LEIVA OLIVENCIA, J.J. La participación de las familias inmigrantes en la escuela intercultural: un estudio cualitativo. Educación y Futuro Digital, 2. Recuperado de <http://www.cesdonbosco.com/revista/articulos2010/noviembre10/leiva.pdf 2011>.

MASSOT, I., DORIO, I. y SABARIEGO, M. Estrategias de recogida y análisis de información. En BISQUERRA, R. (coord.). Metodología de la investigación educativa. pp. 329-366. Madrid: La Muralla. 2004

MESA, M.T., y SOLBES, V. Buenas prácticas en educación intercultural y mejora de la convivencia. Wolters Kluwer España. 2012

MINISTERIO DE TRABAJO e INMIGRACIÓN. Colección de Documentos del Observatorio Permanente, 2009 Recuperado de http://www.mtin.es

MINISTERIO DE TRABAJO E INMIGRACIÓN. La integración del alumnado extranjero en la escuela. Monografías Foro. 2008 Recuperado de $<$ http://www.foroinmigracion.es/es/MANDATO-FORO-2006 2010/Informes/docs/INTEGRACION_ALUMNADO_EXTRANJERO_2008.pdf>.

NÍKLEVA, D.G. EI alumnado extranjero en el marco de la educación

inclusiva. Tejuelo: Didáctica de la Lengua y la Literatura. Educación, (15), 38-48. 2012

PAJARES, M. Inmigración y mercado de trabajo. Informe 2010. Madrid: Ministerio de Trabajo e Inmigración. (2010). Recuperado de

$<$ http://extranjeros.mtin.es/es/ObservatorioPermanenteInmigracion/Publicaciones/conte nido_0004.html>.

PONS, S.C., ROVIRA, J.P., y PEDRO, L.N. A propósito de la acogida de alumnado extranjero. Paradojas de la educación inclusiva en Cataluña (España). Revista latinoamericana de educación inclusiva, 6(1), 105-122. 2012

REAL DECRETO 2393/2004, DE 30 DE DICIEMBRE, por el que se aprueba el reglamento de la Ley Orgánica 4/2000, de 11 enero, sobre Derechos y Libertades de los extranjeros en España y su integración social (BOE núm. 6, de 7 de enero de 2005. Corrección de errores en BOE núm. 130, de 1 de junio), 2011. Madrid. España. Recuperado en: http://www.mir.es/SGACAVT/derecho/rd/rd2393_2004.html

SANTOS REGO, M.A. Las familias inmigrantes en la escuela. ¿Cómo avanzar en su integración desde una perspectiva socioeducativa? En GARCÍA, J. y LACOMBA, (Eds.). La inmigración en la sociedad española. Barcelona: Ediciones Bellaterra, pp. 483-505. 2008

SANTOS REGO, M.A., y LORENZO MOLEDO, M.D.M. La participación de las familias inmigrantes en la escuela: Un estudio centrado en la procedencia. Revista De Educación, (350), 277-300. 2009

STAKE, R.E. Investigación con estudio de casos. Madrid, Morata. 2005 
SOCIAS, C., BALLESTER, L., y MARCH, M. El enfoque de la competencia familiar, una experiencia de trabajo socioeducativo con familias. Pedagogía social. Revista interuniversitaria, (21), 13-37. 2013

TERRÉN, E. y CARRASCO, C. Familia, Escuela e Inmigración. Migraciones (22), 946. 2007

YESTE, C.G., CASABONA, N.M., y COMPTE, M.S. La Implicación y la Participación de las Familias: Elementos Clave para la Mejora del Aprendizaje en los Centros Educativos con Alumnado Inmigrante. Indivisa: Boletín de estudios e investigación, (13), 192-212. 2013.

\section{Como referenciar este artigo}

GARCIA CARMONA, Marina.; CONDE LACÁRCEL, Alfonso. Estudio de caso sobre la integración socio-educativa de familias inmigrantes en Granada (España). Revista Ibero-Americana de Estudos em Educação, Araraquara/SP, v. 11, n. 3, p.1073-1090, 2016. Disponível em: <https://dx.doi.org/10.21723/riaee.v11.n3.8939>. E-ISSN: 19825587.

Submetido em: 01/06/2016

Aprovado em: 29/07/2016 